\title{
HSPB8 promotes cancer cell growth by activating the ERK-CREB pathway and is indicative of a poor prognosis in gastric cancer patients
}

\author{
JIAN SHEN $^{1}$, MINZHE LI $^{1}$ and LI MIN ${ }^{2}$ \\ ${ }^{1}$ Department of Surgery, Beijing Chaoyang Hospital, Capital Medical University, Beijing 100020; \\ ${ }^{2}$ Department of Gastroenterology, Beijing Friendship Hospital, Capital Medical University, \\ Beijing Key Laboratory for Precancerous Lesion of Digestive Diseases, \\ National Clinical Research Center for Digestive Diseases, Beijing 100050, P.R. China
}

Received October 26, 2017; Accepted April 11, 2018

DOI: 10.3892/or.2018.6376

\begin{abstract}
Gastric cancer (GC) is one of the most commonly diagnosed malignancies worldwide, especially in East Asia. Discovery of new biomarker and the elucidation of the molecular mechanisms involved in GC development and progression continue to be important issues for both researchers and clinicians. In the present study, we report that siRNA knockdown of heat shock protein family B (small) member 8 (HSPB8) inhibited the proliferation of GC cells and promoted their apoptosis. Analysis of TCGA dataset indicated that the HSPB8 expression level was strongly positively correlated with the KEGG MAPK signaling pathway $(\mathrm{P}<0.001, \mathrm{FDR}=0.006)$ and BIOCARTA CREB pathway $(\mathrm{P}=0.006$, FDR=0.043). The association between HSPB8 and the ERK-CREB pathway was confirmed by western blot analysis and we found that $\mathrm{pERK}$ and pCREB were significantly decreased following HSPB8 knockdown. Downstream genes of the ERK-CREB pathway were all significantly decreased following HSPB8 knockdown. By evaluating the survival of TCGA GC patients, we found that patients with a high HSPB8 level exhibited significantly worse prognosis than those with low HSPB8 in both overall survival (OS) (log-rank $\left.\chi^{2}=10.60, \mathrm{P}=0.001\right)$ and disease-free survival (DFS) (log-rank $\left.\chi^{2}=11.31, \mathrm{P}<0.001\right)$. The methylation level of HSPB8 DNA was significantly negatively associated with its expression $(\mathrm{R}=-0.1368, \mathrm{P}=0.041)$, and positively
\end{abstract}

Correspondence to: Dr Li Min, Department of Gastroenterology, Beijing Friendship Hospital, Capital Medical University, 95 Yong'an Road, Xicheng, Beijing 100050, P.R. China

E-mail: minli@ccmu.edu.cn

Dr Jian Shen, Department of Surgery, Beijing Chaoyang Hospital, Capital Medical University, 5 Gongtinan Road, Chaoyang, Beijing 100050, P.R. China

E-mail: 410078@163.com

Key words: HSPB8, gastric cancer, cell growth, apoptosis, prognosis associated with OS ( $\left.\log -\mathrm{rank} \chi^{2}=10.60, \mathrm{P}=0.001\right)$. In conclusion, we provide evidence that HSPB8 promotes cancer cell growth by activating the ERK-CREB pathway and may serve as a potential prognostic factor in GC patients.

\section{Introduction}

Gastric cancer (GC) is one of the most aggressive cancer worldwide, with approximately 951,600 new cases and 723,100 new deaths in 2012 (1). Even though the global GC incidence is decreasing, the overall disease burden still ranks 5 th in incidence and 3rd in mortality (1). In particular, $40 \%$ of all GC cases occur in China and other East Asian countries, which may be associated with various unhealthy living habits (2). Recently, endoscopy examination is the main approach for GC screening, but the high medical cost of endoscopy and the shortage of endoscopic professionals in primary medical centers largely hampers the early diagnosis of $\operatorname{GC}(3,4)$. Advances in the identification of biomarkers for detecting precancerous and cancerous gastric lesions have offered alternative strategies for GC screening (5). For example, serum levels of pepsinogen I and II have been used in the identification of high GC risk individuals before endoscopic examination in numerous studies $(5,6)$.

$\mathrm{GC}$ is considered as a disease with high heterogeneity, yet the majority of GC patients are treated with similar chemotherapeutic drugs and surgical techniques, resulting in unfavorable combined sequelae and side effects $(7,8)$. Biomarkers also hold high expectation in the preoperative classification of GC. Actually, protein expression levels, non-coding RNAs, gene copy variations, and single nucleotide polymorphisms (SNPs) are all potential biomarkers of GC (9-11). For example, high expression of serpin A1 was found to be an indicator of a poor prognosis (12), and rs629367 was identified as correlated with poor survival in GC patients (13). However, apart from the well-known HER2 amplification, there are few biomarkers that can be utilized in GC patient classification (14). It is still urgent to discover new biomarkers to facilitate clinical decision-making and avoid unnecessary over-treatment. 
Heat shock protein family B (small) member 8 (HSPB8) is a member of the small heat shock protein superfamily, which contains a conservative $\alpha$-crystallin domain at the C-terminal (15-17). The most well-known function of HSPB8 is acting as a chaperone in association with Bag3 in the regulation of macroautophagy $(15,18-20)$. HSPB8 was found to be associated with many diseases, such as cardiomyopathy (21), amyotrophic lateral sclerosis (22) and Alzheimer's disease (23). HSPB8 was also found to be associated with estrogen-related cancers. Piccolella et al found that HSPB8 modulates the proliferation and migration of breast cancer cells (24). Suzuki et al found that HSPB8 regulates TGF- $\alpha$-induced ovarian cancer cell migration (25). However, there are few reports concerning the role of HSPB8 in gastrointestinal cancers. Based on our previous study of the molecular signature of GC subtypes, HSPB8 was found to be involved in both diffuse and intestinal GCs (26). In the present study, we conducted a series of further assays in vitro and in silico to reveal the biological role and potential prognostic value of HSPB8 in GC.

\section{Materials and methods}

Cell line culture and siRNA transfection. Human gastric cancer cell lines (AGS, BGC-803, BGC-823, SCG-7901 and N87) and normal gastric cell line GES-1 were provided by the Cancer Institute and Hospital, Chinese Academy of Medical Sciences (Beijing, China) and maintained by our laboratory. All cells were maintained in Dulbecco's modified Eagle's medium (DMEM) with $10 \%$ fetal bovine serum (FBS) (purchased from Gibco (Grand Island, NJ, USA), in an incubator containing $5 \% \mathrm{CO}_{2}$ at $37^{\circ} \mathrm{C}$. A total of $4 \times 10^{5}$ cells were seeded in each well of 6 -well plates. After culturing for $24 \mathrm{~h}$, cells were transfected with the siRNAs using Lipofectamine 2000 (Life Technologies; Thermo Fisher Scientific, Inc., Waltham, MA, USA) according to the manufacturer's protocol. All siRNAs were de novo synthesized by Gene Pharma Co. (Shanghai, China) and all the sequences are listed in Table I.

Western blot analysis and antibodies. Rabbit antibodies against HSPB8 (1:500 diluted; cat. no. ab96837), CREB (1:1,000 diluted; cat. no. ab31387) and pCREB (Ser113, 1:1,000 diluted; cat. no. ab32096) were purchased from Abcam (Cambridge, MA, USA). Rabbit antibodies against GAPDH (1:10,000 diluted; cat. no. 14C10), Erk (1:2,000 diluted; cat. no. 137F5) and p-Erk1/2 (Thr202/Tyr204; 1:1,000 diluted; cat. no. D13.14.4E) were purchased from Cell Signaling Technology, Inc. (Berkley, MA, USA). Proteins were extracted using lysis buffer (50 mM Tris-HCl, $\mathrm{pH}$ 7.4; 10 mM EDTA; $0.5 \%$ NP-40; $1 \%$ Triton X-100) with a protease inhibitor cocktail (Sigma-Aldrich; Merck KGaA, Darmstadt, Germany) added. SDS-polyacrylamide gel electrophoresis (PAGE) was performed and then proteins were transferred from gels to PVDF membranes. The membranes were incubated with primary antibodies overnight at $4^{\circ} \mathrm{C}$, washed with TBST for 5 times, 5 min each time, and then incubated for $45 \mathrm{~min}$ with HRP-labeled goat anti-rabbit IgG antibodies (1:2,000 diluted; cat. no. ZDR-5306; ZSGB-Bio Co., Beijing, China) at room temperature, washed with TBST for 3 times, 5 min each time. All proteins were detected with an ECL Plus system (Beyotime Institute of Biotechnology, Jiangsu, China).
Cell proliferation and apoptosis assays. Cells were seeded in 96 -well plates at a density of $2 \times 10^{3}$ cells per well. The viability of cells was determined using Cell Counting Kit-8 (CCK-8; Dojindo Molecular Technologies, Inc., Kumamoto, Japan). The optical density at $490 \mathrm{~nm}$ was measured by Smartspec Model 450 (Bio-Rad Laboratories, Inc., Hercules, CA, USA) after $0,24,48$ and $72 \mathrm{~h}$, in triplicate. The apoptosis rate of the cells was measured by flow cytometry. Cells were obtained and washed with pre-cooling PBS, and re-suspended in binding buffer. Annexin V-FITC $(5 \mu \mathrm{l})$ and 7AAD $(5 \mu \mathrm{l})$ reagents were added to each sample and incubated at $25^{\circ} \mathrm{C}$ for $15 \mathrm{~min}$ away from light. An additional $400 \mu 1$ binding buffer was added and then the cells were analyzed by a flow cytometer (BD Biosciences, Franklin Lakes, NJ, USA). All assays were repeated three times independently.

Reverse transcription and real-time quantitative PCR. Total RNA was extracted using TRIzol reagents and reverse transcripted using SuperScript II reverse transcriptase (all from Life Technologies; Thermo Fisher Scientific, Inc., Waltham, MA, USA) according to the manufacturer's protocol. Real-time quantitative PCR (qPCR) was conducted in triplicates using Applied Biosystems 7500 using the SYBR-Green PCR Master Mix (Roche). Relative mRNA levels of each gene were normalized to GAPDH. All primer sequences used in this study are listed in Table II.

Online patient data acquisition and statistical analysis. Phenotype data with genetic information of the patients were all downloaded from TCGA (http://cancergenome.nih. gov/) (27,28). For Gene Set Enrichment Analysis (GSEA) (29), a Pearson correlation based method (1000 permutations of phenotype were run as recommended) was applied to evaluate the correlation between HSPB8 expression and gene sets of Kyoto Encyclopedia of Genes and Genomes (KEGG) (30) and BIOCARTA (31). A FDR q-value $<0.25$ was considered significant. For survival analysis, the median level of mRNA/methylation of HSPB8 was chosen as the cut-off to separate two subgroups and the Log-rank test was applied and corresponding Kaplan-Meier plots were drawn to show the results intuitionally. Correlation between mRNA and methylation of HSPB8 was calculated by Pearson Chi-square analysis. For cell proliferation, apoptosis and gene expression analysis, unpaired Student's t-tests were used to compare two groups when the means follow the normal distribution, and $\mathrm{P}<0.05$ was considered statistically significant.

\section{Results}

Knockdown of HSPB8 inhibits gastric cancer cell proliferation. To evaluate the expression of HSPB8 in GC cells and normal gastric epithelial cells, we detected HSPB8 in five GC cell lines (AGS, BGC-803, BGC-823, SGC-7901 and N87) and one gastric epithelial cell line (GES-1). The results demonstrated that HSPB8 was relatively higher in the GC cells than that noted in the normal epithelial cells (Fig. 1A). To examine the proliferation-promoting potential of HSPB8, two siRNAs targeting HSPB8 were designed (sequences are shown in Table I) and transfected into AGS and BGC-803 cells. The HSPB8 protein levels were decreased by both siRNAs 
A

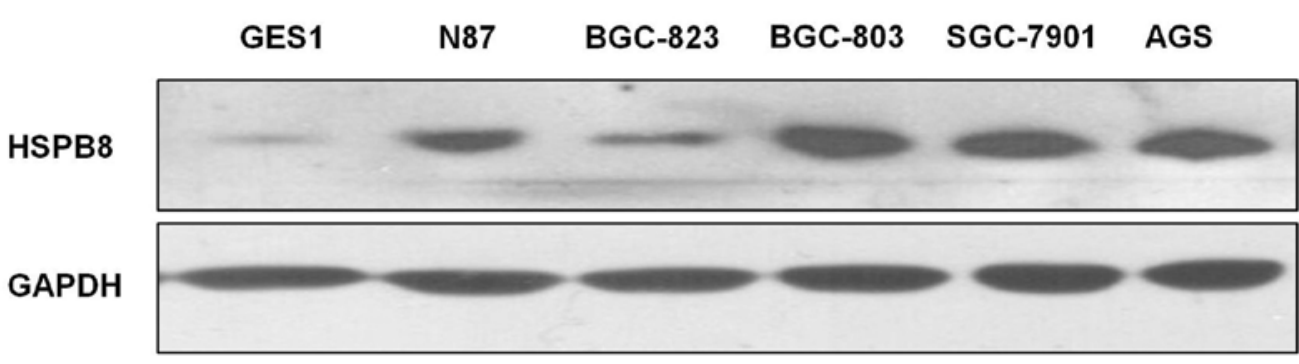

B

AGS cells

HSPB8

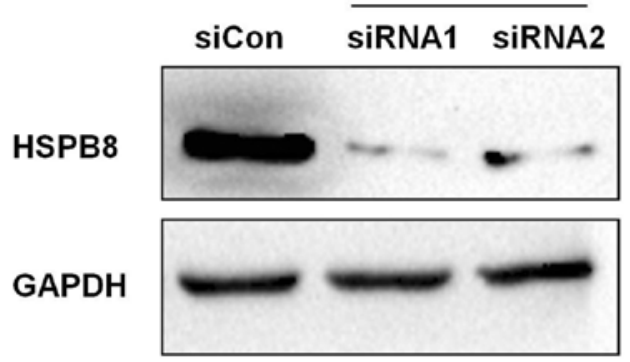

D

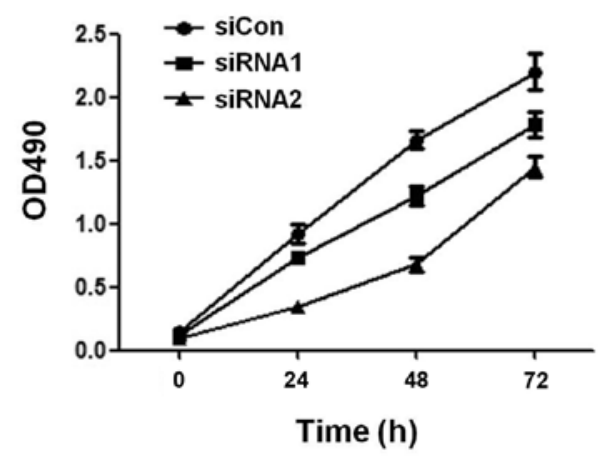

$\mathbf{F}$

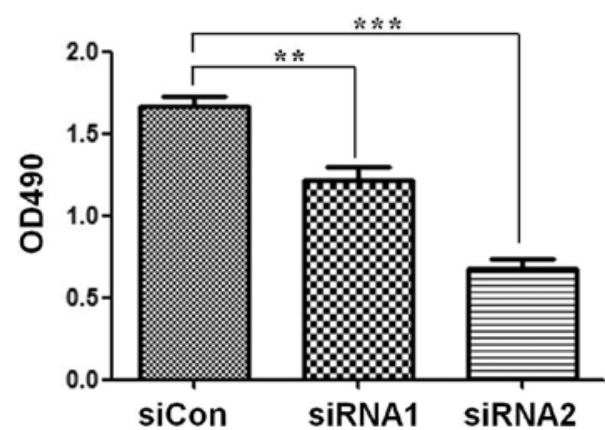

C

BGC-803 cells

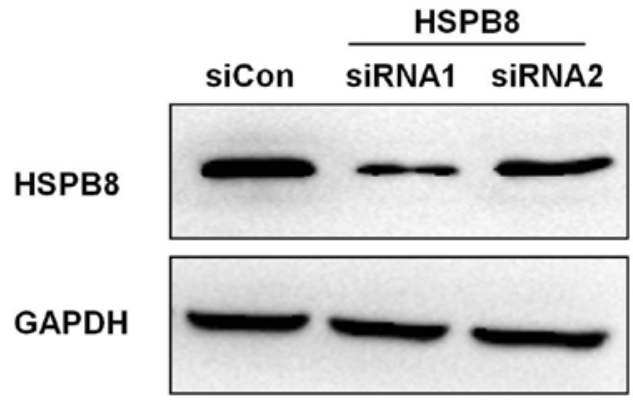

E

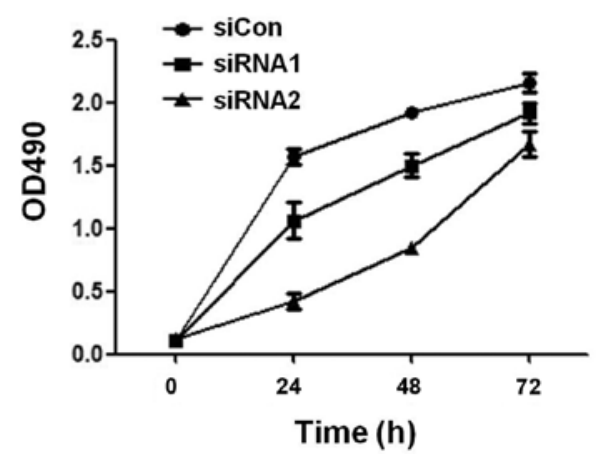

G

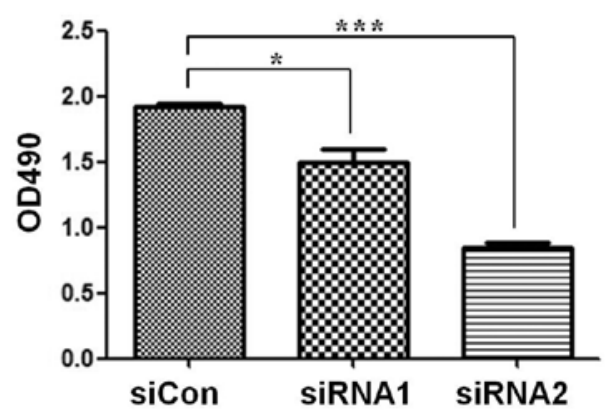

Figure 1. Efficiency of HSPB8 siRNA knockdown and its effect on gastric cancer cell proliferation. (A) HSPB8 expression was higher in five GC cell lines (AGS, BGC-803, BGC-823, SGC-7901 and N87) than the level in a gastric epithelial cell line (GES-1). (B) Efficiency of siRNA knockdown at the HSPB8 protein level in AGS cells. (C) Efficiency of siRNA knockdown at the HSPB8 protein level in BGC-803 cells. (D) Proliferation curves of AGS cells with siRNA knockdown of HSPB8 as determined by CCK-8 assay. (E) Proliferation curves of BGC-803 cells with siRNA knockdown of HSPB8 as determined by CCK-8 assays. (F) OD490 levels of AGS cells transfected with HSPB8-siRNA1 and -siRNA2 and control AGS cells at 48 h. (G) OD490 levels of BGC803 cells transfected with HSPB8-siRNA1 and -siRNA2 and control BGC803 cells at $48 \mathrm{~h}$. Mean \pm SD of three independent experiments were displayed with the Student's t-test to calculate the statistical significance. ${ }^{*} \mathrm{P}<0.05,{ }^{* *} \mathrm{P}<0.01,{ }^{* * *} \mathrm{P}<0.001$. HSPB8, heat shock protein family B (small) member 8 .

Table I. Sequences of the siRNAs used in the present study.

HSPB8 SiRNA1

HSPB8 SiRNA2

NC siRNA
TGAAGTTCTTAGAAACAATGCTT TGTTGAGTAAGGAGGGACCTT ACGUGACACGUUCGGAGAATT 
A

AGS cells

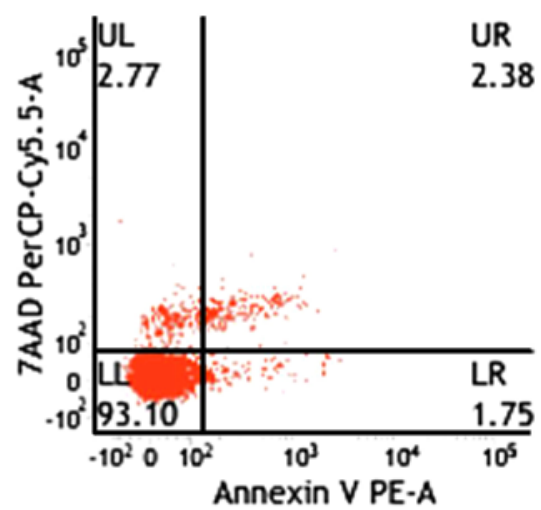

B

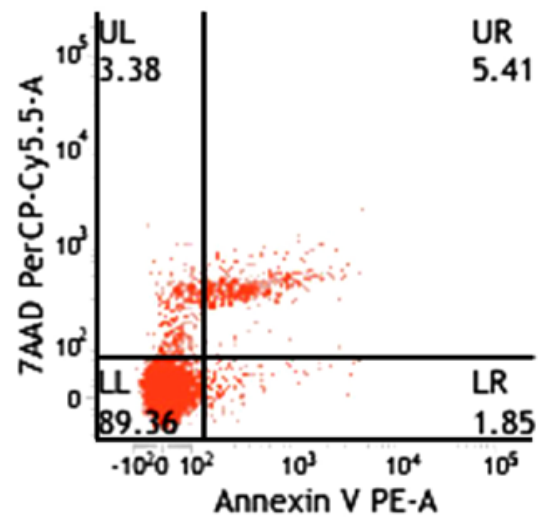

C

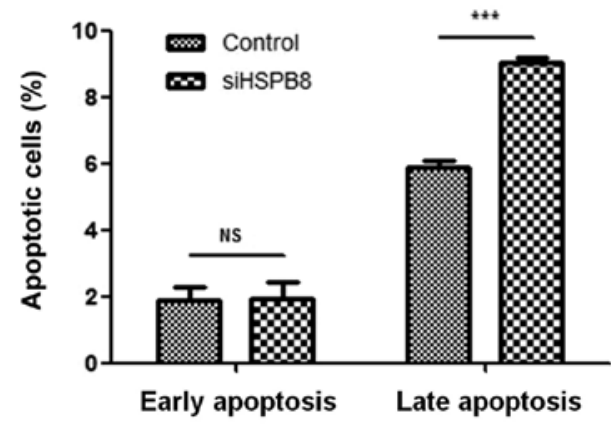

D

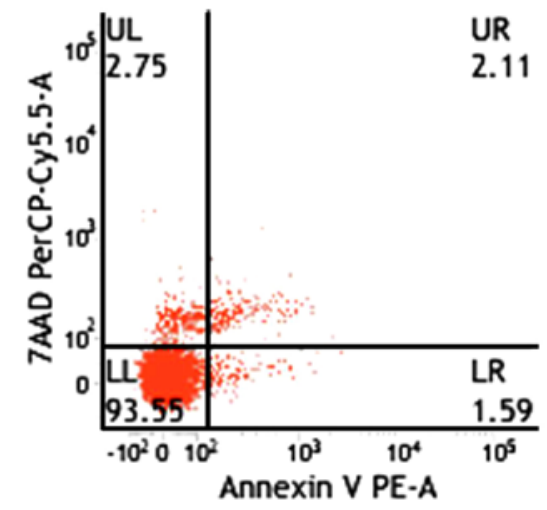

E

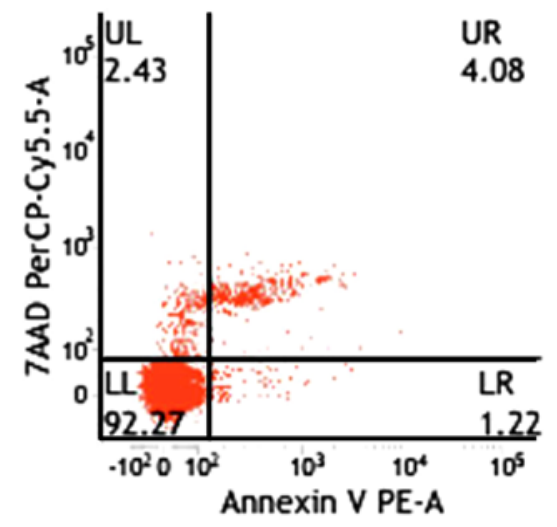

$\mathbf{F}$

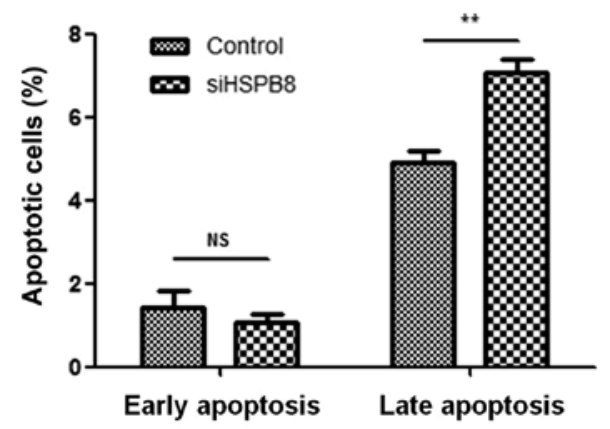

Figure 2. Effects of HSPB8 knockdown on gastric cancer cell apoptosis. Apoptosis levels were determined by flow cytometric analysis with Annexin V and 7AAD staining, and all experiments were triplicated. Representative flow cytometric charts of (A) control AGS cells and (B) HSPB8-knockdown AGS cells. (C) Percentages of early and late apoptotic cells in the control and HSPB8-knockdown AGS cells. Representative flow cytometric charts of (D) control BGC-803 cells and (E) HSPB8-knockdown BGC-803 cells. (F) Percentages of early and late apoptotic cells in control and HSPB8-knockdown BGC-803 cells. ${ }^{* *} \mathrm{P}<0.01,{ }^{* * * *} \mathrm{P}<0.001$; NS, not significant at $\mathrm{P}>0.05$. HSPB8, heat shock protein family $\mathrm{B}$ (small) member 8 .

successfully (Fig. 1B and C), and the proliferation rates of both AGS (Fig. 1D and F) and BGC-803 (Fig. 1E and G) cell lines were significantly hampered by HSPB8 knockdown. Considering that the efficiency of siRNA1 was higher than that of siRNA2, the subsequent analyses were all performed using siRNA1.

Knockdown of HSPB8 promotes gastric cancer cell apoptosis. Suppression of cancer cell growth is usually associated with activation of cellular apoptosis. To examine the influence on apoptosis of HSPB8 knockdown, AGS and BGC803 cells transfected with HSPB8-siRNA1 and NC were subjected to Annexin V-FITC/7AAD staining and flow cytometry.
The results indicated that in both AGS (Fig. 2A-C) and BGC-803 (Fig. 2D-F) cell lines, HSPB8 knockdown had no significant influence on early apoptosis but strongly increased the percentage of late apoptotic cells.

HSPB8 is positively correlated with the ERK-CREB pathway. To further reveal the potential mechanism attributed to the oncogenic role of HSPB8 in gastric cancer, the TCGA dataset of gastric cancer expression data was downloaded and analyzed. The GSEA analysis revealed that the HSPB8 expression level was strongly positively correlated with the KEGG MAPK signaling pathway $(\mathrm{NES}=2.042, \mathrm{P}<0.001, \mathrm{FDR}=0.006$, Fig. 3A) and the BIOCARTA CREB pathway (NES=1.776, 

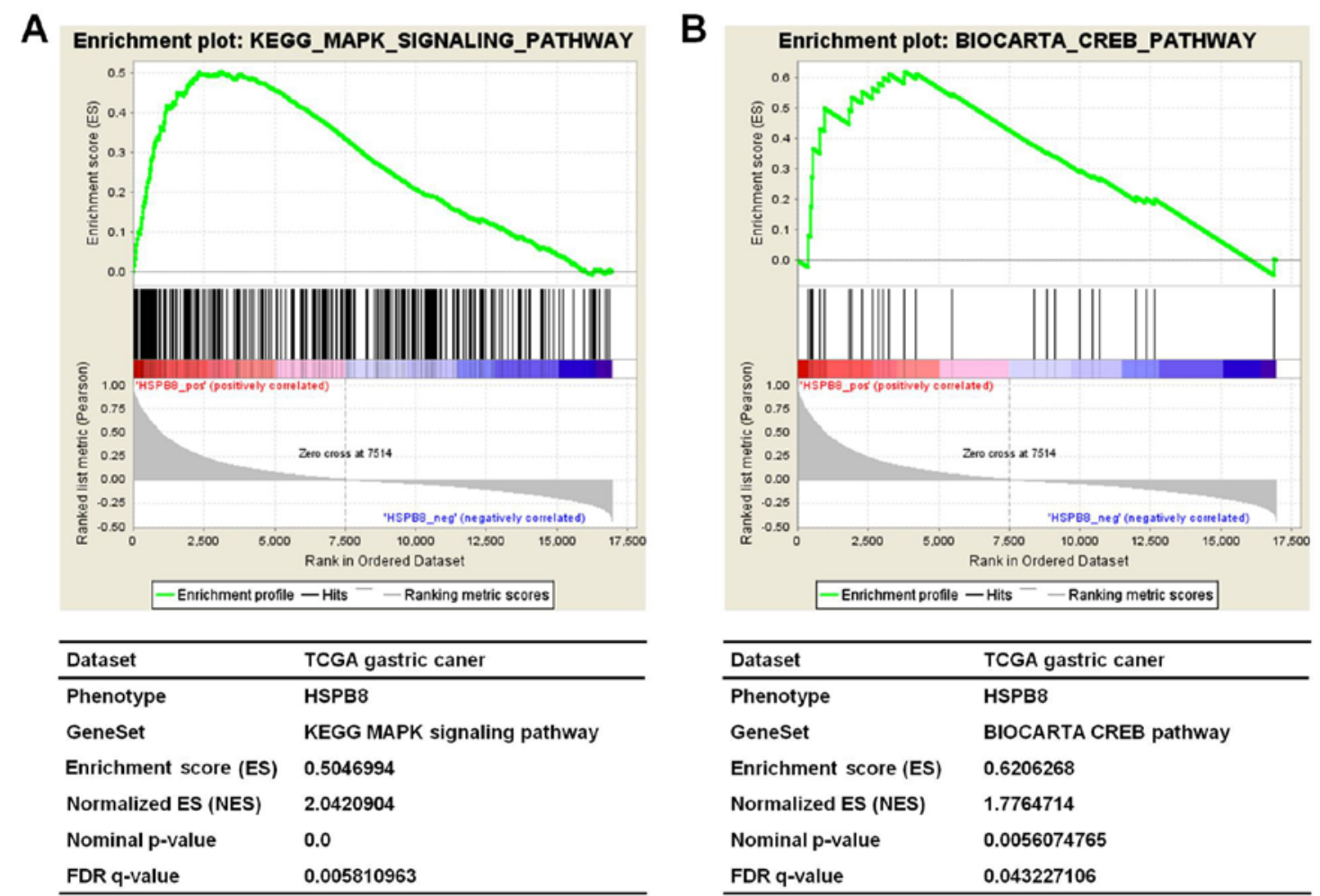

Figure 3. HSPB8 is positively correlated with the MAPK and CREB pathway. TCGA data of 443 gastric cancer patients were used for GSEA analysis. A Pearson correlation based method was adopted and a False Discovery Rate (FDR) $<0.25$ was considered to be statistically significant. (A) GSEA analysis revealed that the KEGG MAPK signaling pathway is positively correlated with HSPB8. (B) GSEA analysis revealed that the BIOCARTA CREB pathway is positively correlated with HSPB8. Upper panels, enrichment plots of GSEA analysis; lower panels, statistical parameters of the GSEA analysis. HSPB8, heat shock protein family B (small) member 8 .

$\mathrm{P}=0.006$, FDR=0.043, Fig. 3B). Activation of the ERK-CREB pathway would be a possible key mechanism of the oncogenic activity of HSPB8.

Knockdown of HSPB8 decreases the activity of the ERK-CREB pathway. To verify our hypothesis of HSPB8's association with the ERK-CREB pathway, we detected levels of phosphorylated and total ERK and CREB proteins under siRNA interference of HSPB8 by western blot analysis. Our results indicated that the total CREB level was stable, while pErk and pCREB were significantly decreased under HSPB8 knockdown (Fig. 4A and B). To reveal whether the changes in pErk and pCREB were time-dependent, we also detected the protein levels of these proteins at 48,72 and $96 \mathrm{~h}$. The level of HSPB8 returned to a normal level at $72 \mathrm{~h}$ after siRNA knockdown, suggesting that HSPB8 is crucial for cell survival and maintains a high synthesis rate in these cells. pErk and pCREB remained suppressed at $96 \mathrm{~h}$ in AGS cells, while in BGC-803 cells the levels of pErk and pCREB were restored at $72 \mathrm{~h}$ (Fig. 4C and D). Downstream genes of the ERK-CREB pathway were also detected by RT-qPCR (primers are shown in Table II), and we found that all the targeted genes we detected were significantly decreased under HSPB8 knockdown (Fig. 4E).

Expression and methylation levels of HSPB8 are potential prognostic factors. To explore whether HSPB8 could be used as a biomarker in gastric cancer, we further evaluated the survival of TCGA gastric cancer patients. When divided by
Table II. Primers for real-time PCR used in the present study.

\begin{tabular}{|c|c|}
\hline Gene name & Primer sequences \\
\hline$H S P B 8$ & $\begin{array}{l}\text { F: CTCCTGCCACTACCCAAGC } \\
\text { R: GGCCAAGAGGCTGTCAAGT }\end{array}$ \\
\hline GAPDH & $\begin{array}{l}\text { F: GGACCTGACCTGCCGTCTAGAA } \\
\text { R: GGTGTCGCTGTTGAAGTCAGAG }\end{array}$ \\
\hline$M M P 9$ & $\begin{array}{l}\text { F: AGACCTGGGCAGATTCCAAAC } \\
\text { R: CGGCAAGTCTTCCGAGTAGT }\end{array}$ \\
\hline EGRI & $\begin{array}{l}\text { F: GGTCAGTGGCCTAGTGAGC } \\
\text { R: GTGCCGCTGAGTAAATGGGA }\end{array}$ \\
\hline CCNAI & $\begin{array}{l}\text { F: GAGGTCCCGATGCTTGTCAG } \\
\text { R: GTTAGCAGCCCTAGCACTGTC }\end{array}$ \\
\hline CCND1 & $\begin{array}{l}\text { F: CGGCAAGTCTTCCGAGTAGT } \\
\text { R: CCTCCTTCTGCACACATTTGAA }\end{array}$ \\
\hline CCN1 & $\begin{array}{l}\text { F: GGATGGTAGTTTTGAGTCACCAC } \\
\text { R: CACGAGGATAGCTCTCATACTGT }\end{array}$ \\
\hline$B C L 2$ & $\begin{array}{l}\text { F: CCTCCTTCTGCACACATTTGAA } \\
\text { R: CGGTTCAGGTACTCAGTCATCC }\end{array}$ \\
\hline
\end{tabular}

F, forward; R, reverse.

the median expression level, the patients with a high HSPB8 level exhibited a significantly worse prognosis than those with 
A

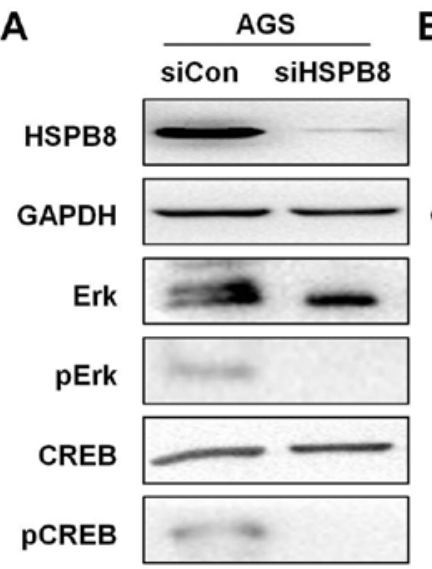

E
B

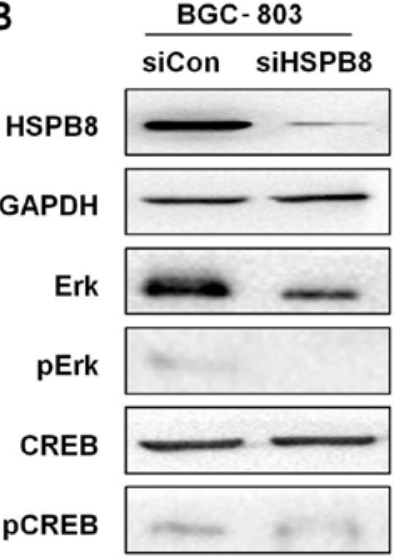

C

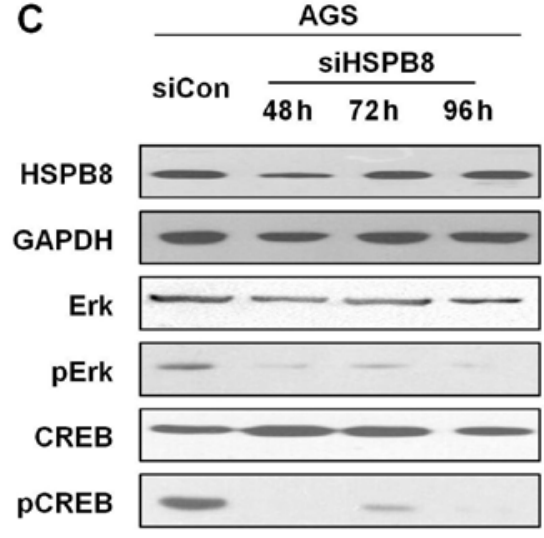

D

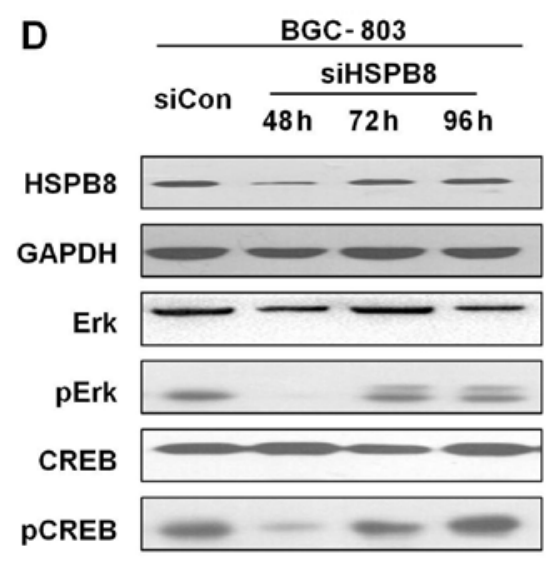

Figure 4. siRNA knockdown of HSPB8 decreases the activity of the ERK-CREB pathway. Protein levels of total and phosphorylated Erk (Erk and pErk) and CREB (CREB and pCREB) in (A) control and HSPB8-knockdown AGS cells and in (B) control and HSPB8-knockdown BGC-803 cells. Protein levels in (C) control and HSPB8-knockdown AGS cells and in (D) control and HSPB8-knockdown BGC-803 cells at different time points. (E) mRNA levels of downstream genes of the ERK-CREB pathway under the siRNA knockdown of HSPB8. Mean \pm SD of three independent experiments were displayed with Student's t-test to calculate the statistical significance. ${ }^{*} \mathrm{P}<0.05,{ }^{* *} \mathrm{P}<0.01,{ }^{* * *} \mathrm{P}<0.001$. HSPB8, heat shock protein family B (small) member 8 .

low HSPB8 in both overall survival (OS) (log-rank $\chi^{2}=10.60$ $\mathrm{P}=0.001$, Fig. 5A) and disease-free survival (DFS) (log-rank $\chi^{2}=11.31, \mathrm{P}<0.001$, Fig. 5B).

DNA methylation data were also co-analyzed with expression data, and indicated that the methylation level of HSPB8 DNA was significantly negatively associated with its expression $(\mathrm{R}=-0.1368, \mathrm{P}=0.041$, Fig. $5 \mathrm{C})$. Patients with high HSPB8 methylation level exhibited a significantly better prognosis than those with low methylation in regards to OS (log-rank $\chi^{2}=10.60, P=0.001$, Fig. 5D).

\section{Discussion}

The small heat shock protein (sHsp) superfamily consists of a series of 15-30 kDa proteins with a common $\alpha$-crystallin domain at the C-terminal. The most well-studied role of $\mathrm{sHsp}$ is acting as molecular chaperones $(32,33)$, i.e. preventing the aggregation of enzymes under heat shock conditions and stabilizing the proteins. There are also numerous studies revealing that sHsp promote the functional refolding of proteins after urea denaturation in an ATP-independent manner (34). There are many members of the sHsp superfamily involved in GC, such as HSP70 (35), HSP110 (36) and HSP27 (37). However, HSPB8 has drawn little attention in this field.
In our previous study, we conducted a pilot investigation of expression-based GC biomarker screening and found that HSPB8 is a potential biomarker in both diffuse and intestinal GC (26). In the present study, our results indicated that HSPB8 is a proliferation-promoting protein in GC cell lines, which could also aid in the evasion of apoptosis by GC cells. The oncogenic role of HSPB8 is in accordance with most other sHsp members, such as HSP70 (35), HSP110 (36) and HSP60 (38).

To reveal the possible mechanism of HSPB8 in GC, we identified molecular pathways associated with HSPB8 expression by analyzing the TCGA GC dataset. From all KEGG and BIOCARTA gene sets, we found that the pathways of MAPK and CREB were positively correlated with HSPB8, which was also validated by western blot analysis in two GC cell lines. Furthermore, we found that the targeted genes of the ERK-CREB pathway (MMP9, EGR1, CCNA1, CCND1, CCN1 and BCL2) were significantly decreased under HSPB8 knockdown. The positive correlation between CCN1 and HSPB8 (39) and the association between BCL2 and HSPB8 (40), were both reported in other cell lines, which may partially verify our finding of HSPB8's role in the regulation of the ERK-CREB pathway.

It is worth noting that HSPB8 was restored to a normal level at $72 \mathrm{~h}$ after siRNA knockdown, suggested that HSPB8 

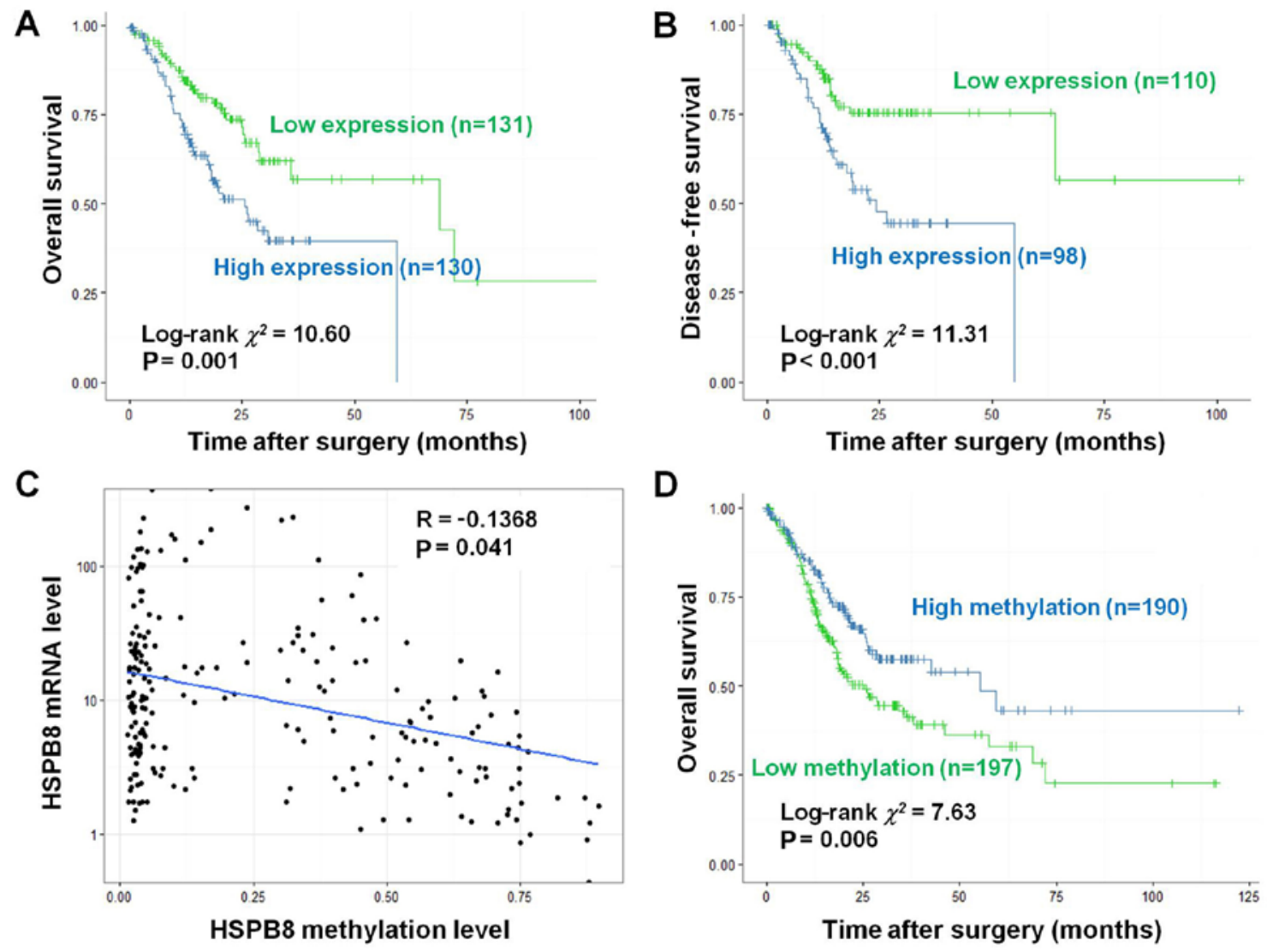

Figure 5. High expression and low methylation levels of HSPB8 are indicative of a poor prognosis in gastric cancer patients. (A) The Kaplan-Meier curve indicated that gastric cancer patients with high expression levels of HSPB8 had shorter overall survival (OS) than those with low HSPB8 expression. (B) The Kaplan-Meier curve indicated that gastric cancer patients with high expression levels of HSPB8 had shorter disease-free survival (DFS) than those with low HSPB8 expression. (C) The Spearman correlation analysis indicated that mRNA expression and promoter methylation levels of HSPB8 were negatively correlated. (D) The Kaplan-Meier curve indicated that gastric cancer patients with low methylation levels of HSPB8 had shorter OS than those with high methylation levels. HSPB8, heat shock protein family B (small) member 8 .

is crucial for cell survival and maintains a high synthesis rate in these cells. However, pErk and pCREB levels were restored along with HSPB8 at $72 \mathrm{~h}$ in BGC-803 cells, but maintained a suppressed level in AGS cells until 96 h. Thus, we suggest that HSPB8 knockdown may have a phenotypic effect on pErk and pCREB, but this appears to be cell line-specific (i.e. this consistent change of HSPB8 and pERK/pCREB was observed for the BGC-803 cell line but not for the AGS cell line at different time-points). It is also possible that HSPB8 knockdown resulted in a more far-reaching effect on AGS cells (i.e. even when HSPB8 was restored quickly after siRNA expired at 72 and 96 h, pErk and pCREB remained suppressed). In all, any phenotypic effects observed after transfection at $72 \mathrm{~h}$ should be interpreted with caution, and further investigation of how HSPB8 promotes pErk and pCREB in a cell line-specific manner is warranted.

Additionally, we also explored the potential of HSPB8 as a prognostic biomarker in GC. Based on our results, both the expression level and the methylation level of HSPB8 could be used as unfavorable prognostic factors. Although the expression level and the methylation level of HSPB8 were significantly correlated, the expression factor still achieved a higher discrimination than the methylation factor in GC patients stratified based on survival data. However, further studies with a larger population are needed before developing reagents for clinical use. In our opinion, the effect of HSPB8 in GC is only partially dependent on its methylation level. Transcriptional factor level and activity, histone modification (methylation and acetylation) and chromatin folding are all possible regulators of HSPB8 expression. For TCGA data analysis, most GC patients had a very low HSPB8 level (Fig. 5C), indicating that the differential expression of HSPB8 was only partially determined by its methylation level.

In conclusion, we demonstrated that HSPB8 could promote the proliferation and inhibit the apoptosis of GC cells by activating ERK-CREB signaling. We also demonstrated that high expression of HSPB8 was indicative of a poor prognosis in GC patients. These findings suggest new evidence to improve our understanding of the mechanisms attributed to the carcinogenesis of GC, and provide insight into the development of patient stratification strategies for GC treatment.

\section{Acknowledgements}

Not applicable.

\section{Funding}

This study was supported by the Funding Program for Excellent Talents of Beijing (2017000021469G212). 


\section{Availability of data and materials}

The datasets used during the present study are available from the corresponding author upon reasonable request.

\section{Authors' contributions}

LM designed the study. JS and ML performed the experiments. JS and LM analyzed the data. JS and LM draft the manuscript. All authors read and approved the manuscript and agree to be accountable for all aspects of the research in ensuring that the accuracy or integrity of any part of the work are appropriately investigated and resolved.

\section{Ethics approval and consent to participate}

This study does not contain any research using human participants or animals performed by any of the authors.

\section{Consent for publication}

Not applicable.

\section{Competing interests}

The authors state that they have no competing interests.

\section{References}

1. Torre LA, Bray F, Siegel RL, Ferlay J, Lortet-Tieulent J and Jemal A: Global cancer statistics, 2012. CA Cancer J Clin 65: 87-108, 2015.

2. Chen W, Zheng R, Baade PD, Zhang S, Zeng H, Bray F, Jemal A, Yu XQ and He J: Cancer statistics in china, 2015. CA Cancer J Clin 66: 115-132, 2016.

3. Choi KS, Jun JK, Suh M, Park B, Noh DK, Song SH, Jung KW, Lee HY, Choi IJ and Park EC: Effect of endoscopy screening on stage at gastric cancer diagnosis: Results of the national cancer screening programme in korea. Br J Cancer 112: 608-612, 2015.

4. Wei WQ, Yang CX, Lu SH, Yang J, Li BY, Lian SY and Qiao YL: Cost-benefit analysis of screening for esophageal and gastric cardiac cancer. Chin J Cancer 30: 213-218, 2011.

5. Yeh JM, Hur C, Ward Z, Schrag D and Goldie SJ: Gastric adenocarcinoma screening and prevention in the era of new biomarker and endoscopic technologies: A cost-effectiveness analysis. Gut 65: 563-574, 2016.

6. den Hoed CM, van Eijck BC, Capelle LG, van Dekken H, Biermann K, Siersema PD and Kuipers EJ: The prevalence of premalignant gastric lesions in asymptomatic patients: Predicting the future incidence of gastric cancer. Eur J Cancer 47: 1211-1218, 2011.

7. Alsina M, Gullo I and Carneiro F: Intratumoral heterogeneity in gastric cancer: A new challenge to face. Ann Oncol 28: 912-913, 2017.

8. Ye P, Zhang M, Fan S, Zhang T, Fu H, Su X, Gavine PR, Liu Q and Yin X: Intra-tumoral heterogeneity of HER2, FGFR2, cMET and ATM in gastric cancer: Optimizing personalized healthcare through innovative pathological and statistical analysis. PLoS One 10: e0143207, 2015.

9. Zhang Y, Yang R, Lian J and Xu H: LncRNA Sox2ot overexpression serves as a poor prognostic biomarker in gastric cancer. Am J Transl Res 8: 5035-5043, 2016.

10. Higaki E, Kuwata T, Nagatsuma AK, Nishida Y, Kinoshita T, Aizawa M, Nitta H, Nagino M and Ochiai A: Gene copy number gain of EGFR is a poor prognostic biomarker in gastric cancer: Evaluation of 855 patients with bright-field dual in situ hybridization (DISH) method. Gastric Cancer 19: 63-73, 2016.

11. Yao YS, Yao RY, Zhuang LK, Qi WW, Lv J, Zhou F, Qiu WS and Yue L: MOR1 expression in gastric cancer: A biomarker associated with poor outcome. Clin Transl Sci 8: 137-142, 2015.
12. Kwon CH, Park HJ, Lee JR, Kim HK, Jeon TY, Jo HJ, Kim DH, Kim GH and Park DY: Serpin peptidase inhibitor clade A member 1 is a biomarker of poor prognosis in gastric cancer. $\mathrm{Br}$ J Cancer 111: 1993-2002, 2014.

13. Xu Q, Dong Q, He C, Liu W, Sun L, Liu J, Xing C, Li X, Wang B and Yuan Y: A new polymorphism biomarker rs629367 associated with increased risk and poor survival of gastric cancer in Chinese by up-regulated miRNA-let-7a expression. PLoS One 9: e95249, 2014

14. Qiu M, Zhou Y, Zhang X, Wang Z, Wang F, Shao J, Lu J, Jin Y, Wei X, Zhang D, et al: Lauren classification combined with HER2 status is a better prognostic factor in Chinese gastric cancer patients. BMC Cancer 14: 823, 2014.

15. Carra S, Seguin SJ, Lambert H and Landry J: HspB8 chaperone activity toward poly $(\mathrm{Q})$-containing proteins depends on its association with Bag3, a stimulator of macroautophagy. J Biol Chem 283: 1437-1444, 2008

16. Hamouda MA, Belhacene N, Puissant A, Colosetti P, Robert G, Jacquel A, Mari B, Auberger P and Luciano F: The small heat shock protein B8 (HSPB8) confers resistance to bortezomib by promoting autophagic removal of misfolded proteins in multiple myeloma cells. Oncotarget 5: 6252-6266, 2014.

17. Ke L, Meijering RA, Hoogstra-Berends F, Mackovicova K, Vos MJ, Van Gelder IC, Henning RH, Kampinga HH and Brundel BJ: HSPB1, HSPB6, HSPB7 and HSPB8 protect against RhoA GTPase-induced remodeling in tachypaced atrial myocytes. PLoS One 6: e20395, 2011.

18. Carra S, Seguin SJ and Landry J: HspB8 and Bag3: A new chaperone complex targeting misfolded proteins to macroautophagy. Autophagy 4: 237-239, 2008.

19. Hishiya A, Salman MN, Carra S, Kampinga HH and Takayama S: BAG3 directly interacts with mutated alphaB-crystallin to suppress its aggregation and toxicity. PLoS One 6: e16828, 2011.

20. Marsh NM, Wareham A, White BG, Miskiewicz EI, Landry J and MacPhee DJ: HSPB8 and the cochaperone BAG3 are highly expressed during the synthetic phase of rat myometrium programming during pregnancy. Biol Reprod 92: 131, 2015.

21. Sanbe A, Daicho T, Mizutani R, Endo T, Miyauchi N, Yamauchi J, Tanonaka K, Glabe C and Tanoue A: Protective effect of geranylgeranylacetone via enhancement of HSPB8 induction in desmin-related cardiomyopathy. PLoS One 4: e5351, 2009.

22. Crippa V, Sau D, Rusmini P, Boncoraglio A, Onesto E, Bolzoni E, Galbiati M, Fontana E, Marino M, Carra S, et al: The small heat shock protein $\mathrm{B} 8$ (HspB8) promotes autophagic removal of misfolded proteins involved in amyotrophic lateral sclerosis (ALS). Hum Mol Genet 19: 3440-3456, 2010.

23. Wilhelmus MM, Boelens WC, Otte-Höller I, Kamps B, Kusters B, Maat-Schieman ML, de Waal RM and Verbeek MM: Small heat shock protein HspB8: Its distribution in alzheimer's disease brains and its inhibition of amyloid-beta protein aggregation and cerebrovascular amyloid-beta toxicity. Acta Neuropathol 111: 139-149, 2006.

24. Piccolella M, Crippa V, Cristofani R, Rusmini P, Galbiati M, Cicardi ME, Meroni M, Ferri N, Morelli FF, Carra S, et al: The small heat shock protein B8 (HSPB8) modulates proliferation and migration of breast cancer cells. Oncotarget 8: 10400-10415, 2017.

25. Suzuki M, Matsushima-Nishiwaki R, Kuroyanagi G, Suzuki N, Takamatsu R, Furui T, Yoshimi N, Kozawa O and Morishige K: Regulation by heat shock protein 22 (HSPB8) of transforming growth factor- $\alpha$-induced ovary cancer cell migration. Arch Biochem Biophys 571: 40-49, 2015.

26. Min L, Zhao Y, Zhu S, Qiu X, Cheng R, Xing J, Shao L, Guo S and Zhang $S$ : Integrated analysis identifies molecular signatures and specific prognostic factors for different gastric cancer subtypes. Transl Oncol 10: 99-107, 2017.

27. Cancer Genome Atlas Network: Comprehensive molecular portraits of human breast tumours. Nature 490: 61-70, 2012.

28. Cancer Genome Atlas Research Network, Weinstein JN, Collisson EA, Mills GB, Shaw KR, Ozenberger BA, Ellrott K, Shmulevich I, Sander C and Stuart JM: The cancer genome atlas pan-cancer analysis project. Nature Genet 45: 1113-1120, 2013.

29. Subramanian A, Tamayo P, Mootha VK, Mukherjee S, Ebert BL, Gillette MA, Paulovich A, Pomeroy SL, Golub TR, Lander ES and Mesirov JP: Gene set enrichment analysis: A knowledge-based approach for interpreting genome-wide expression profiles. Proc Natl Acad Sci USA 102: 15545-15550, 2005.

30. Kanehisa M and Goto S: KEGG: Kyoto encyclopedia of genes and genomes. Nucleic Acids Res 28: 27-30, 2000. 
31. Nishimura D: BioCarta. Biotech Software \& Internet Report 2 : $117-120,2004$

32. Shimizu M, Tanaka M and Atomi Y: Small heat shock protein $\alpha \mathrm{B}$-crystallin controls shape and adhesion of glioma and myoblast cells in the absence of stress. PLoS One 11: e0168136, 2016.

33. Liu J, Luo Z, Zhang L, Wang L, Nie Q, Wang ZF, Huang Z, $\mathrm{Hu} \mathrm{X}$, Gong L, Arrigo AP, et al: The small heat shock protein $\alpha$ A-crystallin negatively regulates pancreatic tumorigenesis. Oncotarget 7: 65808-65824, 2016.

34. Nakamoto H, Fujita K, Ohtaki A, Watanabe S, Narumi S, Maruyama T, Suenaga E, Misono TS, Kumar PK, Goloubinoff P and Yoshikawa H: Physical interaction between bacterial heat shock protein (Hsp) 90 and Hsp70 chaperones mediates their cooperative action to refold denatured proteins. J Biol Chem 289: 6110-6119, 2014

35. Bodoor K, Jalboush SA, Matalka I, Abu-Sheikha A, Waq RA Ebwaini H, Abu-Awad A, Fayyad L, Al-Arjat J and Haddad Y: Heat shock protein association with clinico-pathological characteristics of gastric cancer in jordan: HSP70 is predictive of poor prognosis. Asian Pac J Cancer Prev 17: 3929-3937, 2016.
36. Kimura A, Ogata K, Altan B, Yokobori T, Ide M, Mochiki E, Toyomasu Y, Kogure N, Yanoma T, Suzuki M, et al: Nuclear heat shock protein 110 expression is associated with poor prognosis and chemotherapy resistance in gastric cancer. Oncotarget 7: $18415-18423,2016$

37. Giaginis C, Daskalopoulou SS, Vgenopoulou S, Sfiniadakis I, Kouraklis G and Theocharis SE: Heat shock protein-27, -60 and -90 expression in gastric cancer: Association with clinicopathological variables and patient survival. BMC Gastroenterol 9: $14,2009$.

38. Li XS, Xu Q, Fu XY and Luo WS: Heat shock protein 60 overexpression is associated with the progression and prognosis in gastric cancer. PLoS One 9: e107507, 2014.

39. Liu J, Xu W, Sun T, Wang F, Puscheck E, Brigstock D, Wang QT, Davis R and Rappolee DA: Hyperosmolar stress induces global mRNA responses in placental trophoblast stem cells that emulate early post-implantation differentiation. Placenta 30: 66-73, 2009.

40. Kapila N, Sharma A, Kishore A, Sodhi M, Tripathi PK, Mohanty AK and Mukesh M: Impact of heat stress on cellular and transcriptional adaptation of mammary epithelial cells in riverine buffalo (Bubalus bubalis). PLoS One 11: e0157237, 2016. 